\title{
Entre Formulaire et souscription
}

Le conflit de deux radicalités catholiques à Paris en 1661

\section{Alain Cantillon}

\section{(2) OpenEdition}

Journals

Édition électronique

URL : http://journals.openedition.org/assr/22107

DOI : $10.4000 /$ assr.22107

ISSN : 1777-5825

Éditeur

Éditions de l'EHESS

Édition imprimée

Date de publication : 1 juin 2010

Pagination : 19-38

ISBN : 978-2-7132-2254-2

ISSN : 0335-5985

Référence électronique

Alain Cantillon, «Entre Formulaire et souscription», Archives de sciences sociales des religions [En ligne], 150 | avril-juin 2010, mis en ligne le 14 septembre 2010, consulté le 01 mai 2019. URL : http:// journals.openedition.org/assr/22107; DOI : 10.4000/assr.22107 


\section{Alain Cantillon}

\section{Entre Formulaire et souscription Le conflit de deux radicalités catholiques à Paris en 1661}

Le Formulaire est une profession de foi, une formule (on rencontre ces différents termes dans les écrits qui participent à son élaboration) que l'Assemblée générale du clergé de France envoie à l'ensemble des prélats le $1^{\text {er }}$ septembre 1656, puis, transformé, le 17 mars 1657 :

« Je me soûmets sincerement à la Constitution du Pape Innocent X. du 31. mai 1653. Selon son veritable sens, qui a esté determinée (sic) par la Constitution de N.S.P. le Pape Alexandre VII du 16 octobre 1656. Je reconnois que je suis obligé en conscience d'obeyr à ces Constitutions, $\&$ je condemne de cœur et de bouche la doctrine des cinq propositions de Cornelius Jansenius, contenuë dans son livre intitulé, Augustinus, que ces deux Papes \& les Evesques ont condemnée; laquelle doctrine n'est point celle de saint Augustin, que Jansenius a mal expliquée contre le vray sens de ce saint Docteur. » ${ }^{1}$

Comme le précise l'extrait du procès verbal de la séance du 17 mars 1657 de l'Assemblée générale du clergé (publié dans un ouvrage qui constituera l'un des points d'ancrage de la présente étude), l'emploi d'une même formule par tous les prélats (et donc par tous ceux que ces prélats feront souscrire aux constitutions d'Innocent X et d'Alexandre VII sur les cinq propositions) a pour fin de produire de l'uniformité : "afin qu'il y ait uniformité en ces souscriptions, les prélats se serviront de la formule suivante... ${ }^{2}$. Mise en forme donc de l'énonciation de la soumission à une doctrine, et de la condamnation d'une autre doctrine : il n'est pas question d'exprimer une croyance individuelle, mais de professer une adhésion à une croyance commune à toute une Église (Église de France au sein de l'Église romaine).

1. Version de 1657. Relation des deliberations du clergé de France, sur les Constitutions de nos SS. PP. les Papes Innocent X. \& Alexandre VII. (...) A Paris, chez Antoine Vitré, Imprimeur ordinaire du Roy, \& du Clergé de France. M. DC. LXI. avec privilege du Roy, p. 87.

2. Ibid. Dans cette formule, "je me soumets " prend la place de « je souscris " (sur " suscripsi » voir Fraenkel, 1992 : 33-40 "souscriptions et signatures ») Rien ne dit, d'ailleurs, que cette souscription (que la copie manuscrite de la formule) ne doive pas être suivie d'une signature. 
Ce redoublement de l'uniformité - uniformité de doctrine puis uniformité de soumission à cette doctrine uniforme -, constitue le point délicat, le problème dont l'invention d'une formule, du Formulaire, devrait constituer la solution. Le nœud du problème de croyance apparaît ici comme une question d'écriture, d'énonciation écrite : le moment où l'écriture imprimée doit se transmuer en écriture manuscrite, dans la "souscription » :

"il a esté resolu par le consentement general des Provinces: [...] 3. Qu'à (sic) la formule de la souscription que doivent faire toutes les personnes Ecclesiastiques, suivant la deliberation precedente du premier Septembre sera adjoustée à (sic) cette Constitution, afin qu'elle soit souscrite conjointement avec la precedente : Et que les Prelats seront exhortez de faire proceder à cette souscription dans un mois. 4. Afin qu'il y ait uniformité en ces souscriptions, les Prelats se serviront de la formule suivante, $[\ldots] »($ p. $86-87)$.

Le point numéro trois ne peut se comprendre qu'au prix d'une correction, en remplaçant "qu'à " par "que ", mais, même alors, les points trois et le quatre ne peuvent se concilier que si l'on suppose que la "formule suivante ", qui est le Formulaire, est aussi celle dont il est question au début du troisième point, ce qui n'est pas explicite ici. Si l'on prend, au contraire, ce passage tel qu'il est, on voit apparaître un mouvement d'accumulation ou de fouillis de formules, comme si le discours achoppait sur le nœud de la souscription, ou comme s'il le renouait sans cesse, l'épaississant au fil du temps. Ce fait d'énonciation peut s'interpréter de deux façons complémentaires, d'une part, comme l'une des marques du caractère terriblement répétitif et redondant de cet ouvrage, d'autre part, comme l'indication de la nature problématique de la production d'un formulaire et du passage à la souscription. La relation entre radicalités religieuses et action d'écriture est visible avec un relief exceptionnel dans le problème d'énonciation écrite que constitue la souscription d'un formulaire, de ce moment où la lente, difficile, et pourtant progressive venue au jour d'une doctrine unique et uniforme à professer est supposée se transformer en l'événement d'une profession de foi, où un processus engageant tous les pouvoirs institués est censé basculer dans un acte de foi individuel, et où la production officielle de pièces imprimées fortes des puissances légales des autorités ecclésiale et royale devraient faire apparaître leur efficace par la multiplication de souscriptions manuscrites d'actes de soumission individuels comme les actes de foi.

On étudiera concrètement la précipitation de la croyance dans l'écrit en deux temps, tout d'abord sur son premier versant, celui de la production, dans un ouvrage imprimé, d'une croyance comme hérésie, et de la souscription d'une formule de condamnation de cette hérésie comme profession de foi orthodoxe, ensuite - dans deux lettres de Jacqueline Pascal - sur son second versant, celui de la profession de croyance par énonciation écrite manuscrite comme action de résistance à cette souscription. 


\section{Produire un formulaire}

Le livre intitulé Relation des délibérations du clergé de France [...] est un ouvrage composite qui a connu plusieurs éditions, accumulatives, en 1656, 1661 et audelà. On s'occupera d'un exemplaire édité en 1661, conservé à la BNF sous la cote LD5-252, contemporain de la tentative d'imposition de la souscription du Formulaire, et des lettres de Jacqueline Pascal qui serons étudiées dans la seconde partie de cet article.

Cet ouvrage est formé de la succession, sans coupure, de quarante-sept pièces diverses par leur nature et par leur taille, que l'on peut regrouper, selon le principe que l'on retient, en deux, trois ou quatre sections d'importance inégale. L'absence de table des matières, de chapitre, de titres courants, réduit à fort peu de choses les indications de composition. Le doublement de l'extrait du privilège du roi, une première fois page 126 , et une seconde à la fin de la dernière page (152), donne la seule indication qui s'impose sans nécessiter une analyse. Ce qui précède la page 126 concerne les délibérations du clergé de France sur les constitutions des papes et sur les cinq propositions, ce qui la suit ne traite que de la traduction française du missel romain. L'affaire de la traduction du missel constitue une sorte de cas de la lutte contre le jansénisme, c'est-à-dire de la tentative de mise en lumière progressive d'une présence de quelque chose qui serait du jansénisme ${ }^{3}$. Ce missel est censé matérialiser l'existence réelle du jansénisme comme problème de doctrine susceptible de se poser dans chaque diocèse, et manifester le risque de propagation de l'hérésie.

Dans la première partie, les pièces se succèdent, sans signe particulier de regroupement ou de séparation, si ce n'est une ligne en forme de frise, pas toujours la même, et sans qu'apparaisse de système particulier dans ces variations. Une seule fois quelque chose change (p. 97) : l'utilisation d'une ligne plus épaisse que les autres - presque aussi épaisse que celle qui précède la première pièce du recueil, la «relation des délibérations... »-, qui marque une articulation, correspondant à une césure de trois ans, entre mars 1657 et décembre 1660 . Il n’y a pas lieu de distinguer, sur d'éventuelles différences de mode d'énonciation, de représentation, et donc d'action, entre le premier écrit, la « relation », et les autres, comme si cette relation n'était qu'un récit d'histoire qui donnerait à connaître les actions (assemblées et délibérations dans ces assemblées) dont résulteraient les écrits suivants comme autant d'actes juridiques. La " relation » est aussi, sous beaucoup d'aspects, un acte juridique, et les pièces données à sa suite comportent souvent une part importante de narration. Dans ce long ensemble qui précède la coupure de 1660, il importe pourtant de remarquer une séparation, qui n'est pas du tout marquée, entre les assemblées de 1654 à 1656 et l'assemblée de 1657 , postérieure à la rédaction de la "relation », et qui n'est présente dans ce recueil

3. Sur cette affaire, voir Grès-Gayer, 2002 : 53-66. 
que par cinq écrits (douze pages), pour ainsi dire escamotés dans le flux continu d'écrits, puisque la première pièce qui en émane est un extrait de procès-verbal qui se place, sans aucune marque de rupture, à la suite de trois autres, dans une subdivision qui porte comme titre "Extrait [au singulier] du procès verbal de l'assemblée générale du clergé de France tenue au grand couvent des Augustins, ès années 1655 \& 1656 [sic pour 56-57...] ».

$\mathrm{Au}$ sujet de ce livre, on peut faire l'hypothèse qu'il présente une structure scripturaire et bibliographique singulière, formée de cette composition, mais aussi de quelques autres faits énonciatifs, une structure active et agissante, qui prend place, qui intervient, dans un ensemble d'actions qui consiste, entre autres choses, à précipiter la croyance dans l'écriture, et par là, à la faire apparaître et à lui donner une forme visible et une certaine stabilité, à en faire un précipité au sens chimique du terme.

Dans un premier temps, on verra comment ce livre agit sur le temps, sur le droit, sur la légitimité pour produire un radicalisme religieux, qui serait une radicalité d'institution - la radicalité de l'Église catholique de France autour de ce moment qu'on appelle "prise de pourvoir de L. XIV »; puis on tentera de comprendre comment cela concourt à l'élaboration d'un triple précipité, ou d'un précipité ternaire, dans l'ordre, de doctrine, de discipline, et de croyance.

Premièrement, la Relation des délibérations : vingt-cinq pages d'une typographie serrée qui présente une caractéristique importante pour notre propos : une réflexivité redondante, forme narrative d'un grand souci de formalité des pratiques. Le principe de la réflexivité redondante est explicitement posé à deux reprises : une première fois à l'articulation de deux assemblées, celle de 1655 et celle de 1656 (p. 17) ; une seconde fois à la fin de l'assemblée de 1656 (pp. 23-24) :

"Le premier du mois de septembre 1656. l'Assemblée generale, où estoient presens Messeigneurs les Evesques qui étaient en cette ville pour leurs affaires, qu'elle avoit priez pour cet effet, apres avoir esté informée par la lecture de cette Relation, de ce qui avoit esté traité \& conclu aux trois Assemblées precedentes, projetta de prendre avec toute sorte d'exactitude une derniere resolution sur cette matiere. Pour cet effet, elle fit faire la lecture, tant de la Constitution \& des Lettres que ces Assemblées lui avoient escrites, que du Bref du Pape adressé à celle-cy, qui lui fut presenté par Monseigneur l'Evesque de Montpellier, suivant l'ordre qu'il en avoit receü de sa Sainteté. »

La relation raconte donc comment elle fut lue, comment la nouvelle assemblée se fonde comme assemblée générale qui prolonge les précédentes assemblées, non générales celles-ci, en premier lieu dans sa lecture. Elle se présente comme un élément d'un formalisme juridique qui institue une tradition garante de la vérité doctrinale :

«Il a esté aussi ordonné que cette Relation sera mise dans le procez verbal, et imprimée separément avec la Constitution, le Brefs, les Lettres patentes du roy, \& les lettres des Evesques. » 
Représentation valide et légitime des assemblées et de leurs délibérations, la relation est ainsi graduellement produite comme pièce d'archive authentique et publique, dans une première évocation comme transmission orale d'une tradition interne aux assemblées du clergé de France, puis comme écrit manuscrit officiel, enfin comme passage à l'imprimé de cet écrit authentifié. Dans ces deux récits la relation se présente comme un acte parmi d'autres, parfois comme le premier de tous les actes.

Le principe de réflexivité se déploie dans le compte rendu de la journée du 11 juillet 1653, où les façons de procéder sont particulièrement développées :

«Secondement, l'on considera que le Pape faisoit mention dans sa Constitution, de la lettre que luy avoient escrite plusieurs Evesques de France, afin qu'il donnast son jugement sur chacune des cinq Propositions. Ce qui donna lieu d'examiner serieusement les deux sortes de Relations, ou Consultations, que les loix Ecclesiastiques aussi bien que les civiles avoient reçuës dans l'usage. En l'une les Evesques après avoir expliqué les doutes, adjoustoient leur jugement, \& en demandoient la confirmation au Pape, comme firent les Evesques d'Afrique en la cause de Pelagius. En l'autre sorte de Relation, apres avoir exposé au Pape la chose mise en doute, ils en demandoient la decision, sans que leur jugement fust inséré dans la procedure de la Relation; dont il y a des exemples anciens dans les Decrets des Papes Siticius, Innocent \& Léon, \& en ceux de leurs Successeurs en chaque siecle. Les Evesques de France, quoy qu'assemblez en Concile national, suivirent cet ordre en la consultation qu'ils addressrent au Pape Léon III. qui est dans les Capitulaires de Charlemagne, sur la validité de l'ordination des Prestres, ausquels les corevesques avoient imposé les mains ; \& la response qui declaroit nulle ces Ordinations, fut en suite acceptée par les Evesques assemblez à Ratisbonne en un Concile suivant. Cette pratique est appuyée d'autres exemples qui regardent les matieres de la Foy, $\&$ de la discipline. Ce qui fit qu'on loüa la prudence de ceux qui avoient escrit la lettre de Consultation, puisque les difficultez du temps les avoient empeschez de donner leur premier jugement, en une Assemblée suffisamment remplie d'Evesques. On observa aussi qu'il estoit necessaire de faire entendre à sa Sainteté que les Evesques pouvoient, lors qu'ils le jugeroient à propos, envoyer leurs relations au saint Siège en y adjoustant leur jugement. Ils reconnoissoient neantmoins que la force de l'autorité Apostolique, donnoit à la decision faite sur la relation des Conciles particuliers, le droit d'obliger toute l'Eglise ; comme saint Augustin a remarqué touchant les lettres du Pape Innocent, \& du Pape Zozime.

«Quant à la matiere qui estoit traittée dans la Constitution, elle estoit si connuë à tous ceux de l'Assemblée depuis douze ans qu'elle avoit esté agitée en France, que l'on n'eut point de peine à recognoistre, que la Decision du Pape confirmoit l'ancienne foy de l'Eglise, enseignée par les Conciles, \& par les Peres, \& renouvelée dans le Concile de Trente ; \& qu'un chacun des Evesques estoit obligé de faire publier, \& executer en son Diocese le contenu en la Constitution, \& de punir des peines ordonnées par le Droit contre les heretiques, ceux qui seroient rebelles à cette Decision.

Il fut donc arresté par l'advis unanime de tous, que les Evesques assemblez acceptoient la Bulle, et acquiessoient aux choses decidées avec toute sorte de respect $\&$ de soûmission. Que l'on respondroit au Pape avec des remercimens avec des congratulations, pour les soins que sa Sainteté avoit pris de condemner ces erreurs; en l'asseurant que les Evesques exécuteroient fidellement le contenu en sa Constitution, d'autant plus que le roy leur donnoit sa protection par les Lettres patentes, qu'il avoit fait expedier sur ce sujet, lesquelles enjoignent à tous les Officiers d'assister les Evesques lorsqu'ils 
en seront requis par eux, ou leurs Promoteurs. Il fut aussi arresté que l'on mettrait dans la lettre une clause qui conservast aux Evesques de France le droit de juger en premiere instance des matieres de foy, lorsqu'il leur sembleroit utile pour la Religion, soit en executant les peines de Droit contre les heretiques manifestes ; soit en decidant dans une Assemblée les choses douteuses. »(pp. 7-8).

En distinguant ainsi deux espèces de " relations " ou " consultations ", cette relation en invente une autre, dont elle est l'un des individus singuliers : la relation des délibérations d'une assemblée, qui ne s'adresse pas au pape mais, dans un premier temps, à cette assemblée même, qu'elle soit lue au cours d'une séance pour établir une continuité avec les assemblées précédentes, ou qu'elle prenne place parmi les actes qui peuvent donner à cette assemblée une valeur juridique pour l'avenir, et qui, dans un second temps, se tourne vers les lecteurs indéfinis de l'imprimé. Les modalités énonciatives de ce genre de relation sont telles qu'à certains endroits, et assez fréquemment, les limites entre récit et discours s'estompent au point que le discours rapporté semble bien s'effacer devant un simple discours, qu'aucune composante de l'énonciation ne prend en charge. Ici, entre " en l'une les évêques, après avoir expliqué les doutes, ", et " appuyée d'autres exemples qui regardent les matières de la foi, $\&$ de la discipline " aucune marque ne présente l'exposé historiographique comme la relation de propos tenus dans l'assemblée, ce qui tend à lui conférer la valeur d'un énoncé de doctrine absolu.

Quant aux deux formes de relations mentionnées et évaluées dans ce passage, elles correspondent à plusieurs pièces jalonnant le recueil dans lequel s'entrelacent plusieurs correspondances. Ce sont les lettres envoyées par les assemblées au souverain pontife (Innocent X, puis Alexandre VII) : elles consistent toutes en relations qui informent le pape d'une évolution, d'une situation, d'un état des choses qui nécessite une consultation du pape comme souverain de l'Église universelle. Elles demandent plus qu'une décision doctrinale : une prise de position disciplinaire. Elles reviennent avec insistance sur les nombreux précédents qui, dans l'histoire de l'Église, sont censés autoriser la démarche de l'assemblée.

C'est une position pleinement radicale qui se fait jour dans ces écrits, conforme à l'adage, lui aussi emprunté à la tradition, que les papes ne disent pas de choses nouvelles, mais qu'ils les disent de nouvelles façons (p. 46). Le dogme, selon lequel la tradition de l'Église ne serait que le déploiement dans le temps de la vérité de la révélation divine apportée par la parole de Jésus-Christ, est un des piliers du radicalisme catholique : la doctrine orthodoxe plonge, par la tradition, par l'histoire de l'Église aux racines de la vérité. Un autre dispositif idéologique permet au discours de l'institution de se donner en représentation comme émanation du lieu radical de la vérité : l'existence d'une communication directe et non équivoque entre le pape et le saint Esprit. Ce radicalisme institutionnel constitue l'un des fondements de l'entreprise disciplinaire de mise en ordre et de contrôle d'une institution et d'un territoire. Si nous en croyons le passage que nous venons de lire, l'assemblée des évêques de France consulte le pape parce que c'est à lui, et à lui seul, qu'appartient la souveraineté sur l'Église universelle ; et il revient ensuite aux évêques de faire exécuter ses décisions localement. 
Or, ce livre, en produisant une certaine représentation du déroulement de toute cette affaire, intervient lui-même activement dans ce déroulement. Ainsi, explicitement, ouvertement, les lettres au pape, toute remplies qu'elles sont de marques hyperboliques de soumission, organisent-elles avec un grand soin les rapports entre diverses instances de souveraineté de telle sorte qu'il revienne à l'Église de France, et à elle seule, avec l'appui du pouvoir royal, de faire exécuter les décisions du Saint-Siège apostolique. Voici par exemple la réponse à la constitution Cum occasione (31 mai 1653), qui enjoint aux ordinaires des lieux, et aux inquisiteurs de réprimer les rebelles :

«Estant, comme nous sommes, dans les mesmes sentimens, \& faisant profession de la mesme Foy que les Fideles de ces premiers siecles; Nous prendrons soin de faire publier dans nos Eglises \& dans nos Dioceses la Constitution que vOSTRE SAINTETÉ vient de faire, inspiree par le saint Esprit, \& qui nous a esté mise en main par l'Illustrissime Archevesque d'Athenes son Nonce. Nous employerons toutes nos forces pour la faire observer exactement par les peuples qui sont sous notre conduite, afin de faire voir que nous avons tout le respect $\&$ toute la deference que nous devons avoir pour l'autorité de l'Eglise Romaine, laquelle éclatte à present en la personne d'Innocent X. S'il se trouve des hommes assez temeraires pour contrevenir au Decret de V. S. Nous les punirons des mesmes peines dont les Loix chastient les heretiques, sans avoir aucun esgard à la condition des coupables, selon la teneur de sa Constitution \& du Bref qu'elle nous a addressé : \& nous le ferons avec d'autant plus d'ardeur, qu'en France les Evesques ne partagent ce soin avec personne, parce que l'ancienne coustume de ce Royaume, fondée sur le droit commun, ne permet pas qu'il y ait des Inquisiteurs de la Foy. Certes, nous pouvons asseurer V.S. qu'il n'y aura rien qui nous empesche de faire executer sans aucun delay son Decret Apostolique, puisque la pieté de nostre Roy Tres-Chrestien se joint à nostre Zele, \& le fortifie de son autorité. » (p. 37, lettre au pape du 15 juillet 1653).

Est fermement soulignée une différence essentielle entre la discipline particulière de l'Église de France et la discipline générale de l' "Église romaine »: l'absence d'inquisiteurs. Cette particularité forme l'un des éléments de la structure d'autorité et de pouvoir propre à ce que l'on nomme l'Église gallicane, dans laquelle le "bras séculier " n'est plus un "secours à implorer " ${ }^{4}$, mais bien l'autorité elle-même qui par piété et par zèle est la cause de l'exécution du décret apostolique $^{5}$. Au fil du temps, apparaît comme incidemment à la place des évêques de France (galliarum episcopis lettre au pape du 28 mars 54), une Église gallicane (ecclésia Gallicana, lettre au pape du 20 février 1661), c'est-à-dire une partie de l'Église romaine qui n'est pas désignée par sa simple détermination géographique, mais par une caractérisation nationale qui implique justement un lien particulier avec le pouvoir royal. Il y aurait beaucoup à dire sur la façon dont cet ouvrage organise les relations entre le pouvoir des évêques et celui $\mathrm{du}$ roi, dans le choix du vocabulaire (par exemple; p. 6, le roi « exhorte " et «admoneste » les évêques, mais ne peut rien leur « enjoindre »), ou dans la mise

4. Selon les propres termes de la constitution Cum occasione.

5. Sur l'intrication des affaires "jansénistes " et de la question des traditions gallicanes, dès lors que la position des évêques est en jeu, voir Blet, 1995 : 222-224. 
en scène de quelques saynètes qui impliquent, au Louvre, les représentants de l'assemblée du clergé d'une part et de l'autre Mazarin et le roi, ou dans le quasi oubli des difficultés soulevées par certains parlements, ou dans la publication de la correspondance entre l'assemblée et le roi ; ou encore dans une lettre spirituelle du président de l'assemblée à la reine mère, envoyée le premier septembre 1656 (date d'expédition du Formulaire à tous les évêques), qui accompagne l'envoi de la « relation des délibérations ", dans laquelle il lui est dit précisément ce qu'elle doit croire :

Il est donc raisonnable que Vostre Majesté jouisse du fruict des victoires de l'Eglise, $\&$ que pour en estre nourrie elle croye ces importantes veritez; [...] Vostre Majesté croira, [...] Vostre Majesté remerciera Dieu, MADAME, d'avoir donné aux hommes une loy si sainte, [...] Vostre Majesté, MADAME, sera persuadée [...] Vostre Majesté croira, [...]. (pp. 73-74).

Tout cet ouvrage dit, et surtout effectue à sa manière, en tant que publication officielle de pièces authentiques, la remise en ordre, la sortie des temps de trouble, la reprise en main d'une Église et d'un État. Dès sa première page la Relation des délibérations place l'origine des troubles découlant, dit-elle, de la publication de l'Augustinus, dans les dernières années du règne de Louis XIII. Les années qui suivent sont ensuite évoquées, dans une grande indétermination chronologique, comme ce «temps-là », temps du désordre :

«Les desordres qui estoient survenus en ce temps-là, dans diverses Provinces de la France, osterent le moyen aux Prelats de pouvoir tenir commodément les Conciles, ou Assemblées de cette sorte, \& l'esperance de remedier aux maux par cette voye. Leur zele leur en ouvrit un (sic) autre, conforme à l'ancien usage des Eglises d'Occident $\&$ d'Orient, laquelle est necessaire dans les temps difficiles, qui troublent la liberté de l'Assemblée des Conciles dans les Provinces. Ces Prelats formerent une lettre de Consultation addressée au Pape, afin qu'il condamnast en particulier chacune des cinq Propositions, contenant en abbregé la doctrine extraite du livre de Jansenius, \& inserée dans cette lettre, qui fut presentée à sa Sainteté de leur part. Ils la signerent, \& eurent le soin de faire souscrire à cette Consultation plusieurs Evesques absents, jusqu'au nombre de plus de quatre-vingts, qui suppléerent leur présence par leur souscription, ou par leurs lettres particulieres, suivant l'ordre des Canons. » (p. 4).

Le supplément de l'écriture, et en particulier la représentation par « souscription ", et non pas simplement par signature, fournit donc un moyen d'action par temps de désordre, et pour sortir du trouble. C'est l'invention et la définition des cinq propositions qui constitue l'action d'écriture la plus décisive. C'est seulement par une série d'énonciations écrites qu'une hérésie peut être définie. Ce recueil de pièces officielles prend part à cette action globale, et ne le fait pas en rendant compte, par la publication, du travail d'écriture définitoire, mais en le produisant par une représentation parfaitement ordonnée, conforme au modèle général de la légitimité d'un tel travail (les évêques proposent des doutes, puis le pape décide de l'erreur et de la vérité, éventuellement après que des évêques ont proposé des doutes, et ensuite les évêques reçoivent cette décision et la mettent en 
exécution). Certains troubles, toutefois, dans cette représentation, sont pour nous autant de marques de cette production.

Ainsi la "relation des évêques de France à N.S.P. le pape Innocent X » qui se place aussitôt après la "relation des délibérations » et apparaît, bien qu'elle ne porte aucune date, comme la " consultation » envoyée à Innocent $\mathrm{X}$ en temps de trouble - c'est-à-dire entre la publication de la bulle d'Urbain VIII (In Eminenti, publiée en juin 1643), et la bulle Cum occasione d'Innocent X, exactement dix ans plus tard -, ainsi donc cette consultation se justifie-t-elle d'une façon particulièrement incertaine :

"Vostre Sainteté a estably par un nouveau Decret la verité \& la force de cette Bulle, mais parce que chaque proposition en particulier n'a pas esté notée d'une censure speciale, quelques-uns ont creu, qu'il leur restait encore quelque moyen d'employer leurs chicanes \& leurs fuites. Nous esperons qu'on leur fermera entierement le passage, s'il plaist à Vostre Sainteté, comme nous l'en supplions tres-humblement, définir clairement et distinctement, quel sentiment il faut avoir en cette matiere. C'est pourquoy nous la supplions de vouloir examiner $\&$ donner son jugement clair $\&$ certain sur chacune des propositions qui ensuivent, sur lesquelles la dispute est plus dangereuse $\&$ la contention plus échauffée. » (pp. 25-26).

Or, la bulle d'Urbain VIII ne relève, ne délimite aucune proposition condamnable, mais porte un jugement négatif sur l'Augustinus dans son ensemble, et c'est en partie pour cela qu'elle ouvrit plutôt qu'elle ne ferma le passage aux contentions. Quant aux cinq propositions, elles sont dues à Nicolas Cornet, syndic de la Sorbonne qui en proposa l'examen en juillet 1649, ce qui constitua fort précisément le point de départ d'une forte contention. Dans la relation des évêques de France, présentée publiquement dans ce livre comme celle qui aurait été, à une date indéterminée, envoyée au pape, il semble que l'assemblée des évêques propose au siège apostolique des doutes sur une constitution antérieure alors qu'en fait elle impose, par cette représentation même, une hérésie prédéfinie à la qualification du Saint-Siège.

Une telle action énonciative, qui ne peut être ainsi menée que dans le cadre de ce recueil imprimé, tel que nous l'avons décrit, avec ses redondances et sa progressivité, de consultation en consultation et de Constitution en Constitution, qui sans renoncer traque, cerne, circonscrit, délimite et définit l'hérésie (au point que l'assemblée générale de 1660-1661 est dite, p. 97, " tenue [...] sur le sujet du jansénisme » mot qui n'apparaît pas auparavant dans l'ouvrage), consiste en ce que l'on pourrait appeler une scripturarisation totale de la doctrine, de la discipline, et de la croyance. Rien n'apparaît en effet dans ce livre de toutes les négociations nécessaires à Rome ; comme si tout s'était réglé uniquement par des correspondances.

Le Formulaire de souscription constitue la pointe ultime de cette «scripturarisation "; par lui, ce qui est précipité dans l'écrit c'est un fonctionnement institutionnel qui implique la croyance individuelle par profession de foi écrite de chaque membre de l'institution. Cette ultime action d'écriture est présentée 
comme le terme de fin d'une tradition scripturaire dans laquelle rien de nouveau ne peut se produire, si ce n'est une nouvelle façon de dire, une nouvelle formulation. La production de ce formulaire constitue ainsi une nouvelle action énonciative, jamais attestée dans la tradition ecclésiastique.

Nous pouvons certes admettre que cette nouvelle façon de dire ne dit rien de neuf, qu'elle ne dit rien que de très catholique ; pour autant, elle n'en introduit pas moins une grande nouveauté dans le corps ecclésial en portant à une intensité jamais atteinte encore auparavant le procès de "scripturarisation » de la définition de la bonne et de la mauvaise doctrines, que l'on fixe, uniforme, en l'imprimant, non pas seulement dans des livres, mais dans la conscience de chaque ecclésiastique.

Aussi voyons-nous que le chemin de la définition de l'erreur n'est pas ici celui que présente à plusieurs reprises l'ouvrage de Bruno Neveu, L'Erreur et son juge. Il semblerait bien que ce ne soit pas la définition de la bonne doctrine qui soit première, suivie par la définition doctrinale de l'hérésie puis par un discours, d'application, que serait le discours inquisitorial. Ce qui apparaît dans ce livre c'est que la définition de l'hérésie se fait dans un mouvement de construction et de défense d'une position institutionnelle qui nécessite l'identification, la définition d'une hérésie et, dans le même mouvement, sa destruction, ou tout au moins son confinement et sa marginalisation. De la même façon disparaît la distinction entre l'hérésie absolute et l'hérésie liée à un livre singulier (Neveu, 1993 : 252), séparation dont Neveu lui-même, à la suite de certains des théoriciens dont il commente les ouvrages, mettait quelquefois en doute la validité (id. : 463). Certes, il importe peu d'atteindre l'auteur de l'hérésie, comme personne, voire comme auteur, puisque l'on sait bien que cet auteur n'est jamais le véritable auteur, que ce véritable auteur de l'hérésie est bien connu, depuis longtemps, et qu'il est ailleurs; mais cela ne signifie pas pour autant que l'important soit la doctrine absolute, coupée, donc, de l'énonciation hérétique. Ce qui est terrifiant dans l'hérésie, et l'histoire récente (encore récente en 1661) en a donné de grands exemples, c'est la multiplicité virtuelle de son énonciation, et, dans le cas des ecclésiastiques, surtout de cette forme d'énonciation particulière qu'est une profession. Professer, c'est déclarer hautement, publiquement, modalité énonciative dont la souscription peut constituer une adéquate formalisation.

\section{Ne pas souscrire ; écrire}

«Votre Majesté croira » écrivait le président de l'Assemblée à la reine mère ; tout autant, et avant tout, tous les ecclésiastiques croiront, c'est-à-dire tous se soumettront à la constitution Ad sacram, tous se déclareront catholiques, unanimement, autrement dit uniformément; ou bien ceux qui ne souscriront pas à cette formule de profession de foi professeront par là leur appartenance à l'hérésie janséniste, à cette nouvelle hérésie, à cette hérésie nouvellement et enfin, si l'on en croit ce que dit l'ouvrage Relation des délibérations ..., définie. 
C'est à Port-Royal qu'est associé le problème du Formulaire. En particulier, pour l'année 1661, la tradition des études pascaliennes attribue à la sœur Jacqueline de Sainte-Euphémie Pascal deux très belles lettres (Pascal, 1992 : 1073-1093) ${ }^{6}$ qui posent avec une acuité douloureuse le problème de la souscription individuelle et de la croyance comme soumission. Contrairement au recueil des Relations des délibérations, ces lettres ne sont pas authentifiées par leur publication à ce moment avec ces signes de localisation que sont, à défaut d'un nom d'auteur, le nom de l'imprimeur, et ceux des approbateurs, ou encore indirectement au moyen d'une référence explicite dans un autre imprimé, parfaitement identifiable. Une partie d'entre elles, seulement, sera publiée quatre ans plus tard, lors d'une autre phase de ce que les gens de Port-Royal appellent les persécutions, derechef à propos de la souscription du Formulaire. Le reste ne nous est connu que par le biais de copies manuscrites transmises dans les archives de Port-Royal, et plus précisément dans celles de la famille Pascal. Aussi ne pouvons-nous prétendre étudier le problème de la souscription du Formulaire, tel qu'il aurait pu prendre forme à Port-Royal en 1661, et tout particulièrement sous la plume d'une des religieuses, connue pour sa radicalité et pour ses talents d'écrivaine. Cet objet, ces deux lettres, n'étant pas parfaitement déterminé en tant qu'authentiques lettres écrites par Jacqueline Pascal à propos du Formulaire, mais l'étant, au contraire, en tant que lettres conservées, par plusieurs copies et, partiellement publiées, comme représentations actives ${ }^{7}$, extrêmement complexes, stratifiées, du problème de la souscription alors à Port-Royal, et des actions d'écriture induites par l'injonction à souscrire, et par son rejet.

Comment ces lettres produisent-elles une radicalité religieuse pour l'opposer à l'injonction institutionnelle, et comment, ce faisant, précipitent-elles à leur façon la croyance dans l'écrit, en détournant, contournant ou retournant la souscription ? Quelques éléments d'explication : les grands vicaires du cardinal de Retz, archevêque de Paris en exil, viennent, le 8 juin, de publier un mandement - connu comme le premier mandement des grands vicaires sur la signature - pour accompagner le Formulaire, mandement fort subtil, assez équivoque semble-t-il pour que l'on puisse grâce à lui signer le Formulaire en réservant la question du fait, et par conséquent la condamnation de l'Augustinus. Antoine Arnauld, parmi d'autres, se montre alors favorable à la signature.

Le dispositif énonciatif de ce morceau de correspondance se signale par ses complications et mérite une analyse minutieuse dans la mesure où c'est justement de cela qu'il est question dans ces lettres, de la possibilité d'une authentique écriture personnelle. La première lettre est adressée à la sœur Angélique de SaintJean, nièce d'Antoine Arnauld, le destinataire de la seconde lettre. Elle a le même

6. Le texte que nous suivrons est celui de Jean Mesnard dans son édition des CEuvres complètes de Pascal.

7. Une représentation est un artefact qui produit, comme le dit Marin, des effets de présence. 
âge que Jacqueline Pascal, et exerce les fonctions de sous-prieure du monastère de Paris alors que Jacqueline Pascal est la sous-prieure du monastère des champs. C'est donc une lettre de sœur à sœur, et, pour ainsi dire, de sœur jumelle à sœur jumelle dans un monastère qui est si fortement marqué par sa localisation bifide.

Dans cette structure d'envois, l'adresse à l'alter ego prend la valeur d'un mode de destination particulier, indirect, à l'autre, au "père ", extérieur et, selon la lettre à la sœur, peu accessible aux difficultés que soulèvent alors certaines religieuses. La seconde lettre, lettre au père (Pascal, 1992 : 1091-1093) ${ }^{8}$, établit en effet un protocole de lecture qu'il est indispensable de comprendre pour avoir accès à la signification, où au mode d'action de la première. En premier lieu, la lettre au père produit une représentation du mouvement d'accès à l'écriture de la lettre à la sœur comme une double mission qui est aussi un double moment : Jacqueline Pascal aurait tout d'abord eu un "mouvement intérieur, qui n'est pas une chose bien sûre" (ibid.), une "forte pensée d'écrire "(ibid.), et puis, dans un deuxième temps, reçu l'ordre d'Arnauld sous la forme d'un billet (ibid.) Ce dédoublement et redoublement de la mission fonde et autorise deux fois l'écriture, puisque l'ordre reçu assure le mouvement intérieur. Remarquablement en effet, la structure de ces éléments de correspondance se conforme à cette structure de la mission, et l'ordre reçu n'engage pas à reprendre le premier mouvement et à substituer à la lettre écrite à la sœur d'un premier mouvement une autre lettre à la sœur, ou bien la lettre au père qui répond au billet reçu, mais à achever la lettre à la sœur de première mission avec le surcroît d'assurance ${ }^{9}$ donné par la seconde mission.

Récrire la lettre de première mission, pour la ré-adresser, cela aurait pu demander beaucoup de temps parce que la lettre à la sœur dit justement, indirectement, au père beaucoup de choses qu'il aurait été bien difficile de lui exprimer directement ${ }^{10}$. Le traitement accordé au mandement des grands vicaires est sur ce point des plus révélateurs ; la lettre à la sœur, qui en juge les «termes ambigus », « indignes de la sincérité chrétienne ${ }^{11}$, le présente comme une sorte de piège

8. Le terme d'adresse est bien évidemment "Mon Père ", à treize reprises (seulement dix fois " ma chère sœur ", ou " ma très chère sœur " dans l'autre lettre pourtant trois fois plus longue).

9. Pascal (1992: 1092) : "Je l'ai achevée aujourd'hui avec plus d'assurance depuis votre billet, et je vous l'envoie, mon Père, parce que je n'ai pas pu prendre le temps de la récrire pour vous l'adresser. " La « récrire » et non pas la copier.

10. Nous ne pouvons pas exclure, bien évidemment la possibilité que la lettre à la sœur, avant d'être ré-adressée, ait été, contrairement à ce que déclare la lettre au père, totalement reprise, réécrite, voire que la lettre à la sœur n'ait pas préexisté du tout à l'écriture de la lettre au père et qu'elles aient d'emblée été rédigées comme un diptyque; mais ces hypothèses ne changeant absolument rien au fonctionnement structurel de cette paire de lettres, nous pouvons nous dispenser de les prendre en compte dans notre analyse.

11. Pascal, 1992 : 1081-1082 : "Encore que nous entendions fort bien que l'on prétend que notre signature ne nous demande que le respect, c'est-à-dire le silence pour le fait et la créance pour ce qui est de la foi, ce que nous avons toujours été prêtes de témoigner, nous voyons néanmoins que cela est exprimé en termes ambigus et indignes de la sincérité chrétienne. 
qui risque de contraindre à signer le Formulaire (Pascal, 1992 : 1081-1082); à l'inverse la lettre au père le considère non plus du point de vue de ceux, ou celles, qui sont en mesure d'identifier et de défendre la vérité, mais du plus grand nombre, de cette "infinité de personnes qui se laissent conduire à la boucherie comme des agneaux ", dont il "[met] en sûreté la conscience "(Pascal, 1992: 1092). La double mission, et les deux temps de l'écriture, permettent de déployer une antinomie hors de laquelle la pensée pratique peut continuer à se tenir sans compromission. Le dispositif d'énonciation scripturaire rend ainsi possible un discours d'exposition des pensées qui ne soit pas une demande d'avis, puisque justement c'est le problème de la décision personnelle en conscience qui s'y trouve pleinement développé, mais qui n'exclut pas pour autant les autres, les proches, sœurs, mères ou pères, de la corresponsabilité ${ }^{12}$.

Le partage de la responsabilité d'une conscience prend deux formes : une pieuse précaution, et le déploiement extraordinairement complexe et rigoureux d'un art épistolaire. Le récit rétrospectif d'un moment de la première mission, au début de la lettre au père, celui du passage entre la "forte pensée d'écrire » et l'écriture effective d'une lettre, fait état du souci de ceux qui, par le fait de l'envoi de cette lettre, vont devoir entrer en correspondance, et engager leur responsabilité dans leur réponse et leurs conseils :

Ne sachant à qui je m'adresserais, je jetai les yeux sur me sœur Angélique, à qui j'écrivis dans le moment cette longue lettre, après avoir invoqué Dieu et son SaintEsprit pour ceux qui devaient y répondre (Pascal, 1992 : 1091).

Conformément à la double mission, et au changement d'adresse qu'elle suppose ou implique, le passage à l'écriture est marqué d'une indécision persistante de la destination, puisque le choix de l'adresse - cette sœur sur laquelle on jette les yeux à distance, en franchissant ainsi, en un clin d'œil, la séparation des deux sites de Port-Royal - ne détermine pas pour autant qui doit y répondre. C'est un des aspects du problème du libre arbitre, tel qu'il est construit dans ces deux lettres, dans le tissage serré de deux ordres de conditions, d'une part celles qui ressortissent de la théorie théologique, et selon lesquelles personne ne peut savoir quelles doivent être les suites de ses propres actions, et d'autre part celles qui ressortissent de l'organisation sociale et politique supposément adéquate à cette dogmatique : puisque, d'une part, des deux volontés, celle de Dieu et celle de l'homme, la première est la maîtresse et la dominante ${ }^{13}$, et que d'autre part les

Ainsi la plupart désireraient de tout leur cœur qu'il fût pire, sachant bien qu'il n'en fallait pas espérer dans les temps où nous sommes, un meilleur, parce qu'au moins on le rejetterait avec une entière liberté, au lieu que plusieurs seront comme contraints de le recevoir ».

12. L'importance de la "corresponsabilité » dans la correspondance spirituelle a été soulignée voici peu, à propos de Jérôme Nadal, par Pierre-Antoine Fabre à qui nous empruntons le terme.

13. Selon les termes des Écrits-sur-la-grâce-de-Pascal : "Si, donc on demande pourquoi les hommes sont sauvés ou damnés, on peut en un sens dire que c'est parce que Dieu le veut et en un sens dire que c'est parce que les hommes le veulent. Mais il est question de savoir laquelle de ces deux volontés, savoir de la volonté de Dieu ou de la volonté de l'homme, est la maîtresse, la dominante, la source, le principe et la cause de l'autre. »(Pascal, 1963 : 311). 
règles de correspondance imposées aux religieuses, qui se surajoutent aux usages sociaux des lettres, et aux aléas de tout passage par l'écrit, conduisent plusieurs personnes à prendre connaissance de la lettre, rien ne permet de savoir à l'avance, quel que soit le destinataire choisi, qui entrera dans la correspondance, qui sera exposé aux effets de ce discours, et par conséquent de qui l'auteur de la lettre va devenir responsable. Ce jeu engage avant tout la mère abbesse qui, selon les Constitutions de Port-Royal, doit donner la "permission " d'écrire, et peut «supprimer» les lettres «si elle ne les trouve pas comme il faut ${ }^{14}$.

L'aménagement du lieu de l'énonciation, à la fois par les conditions extérieures - règles du monastère, situation particulière de cette sœur dans la hiérarchie ecclésiastique, et dans les relations de famille ${ }^{15}$ - et, comme nous venons de l'analyser, par l'énonciation elle-même, doit être compris dans la perspective de ce qui est ici en jeu : la possibilité d'un libre arbitre, non pas seulement sous un angle métaphysico-théologique, mais aussi, et avant tout maintenant qu'un formulaire a été mis au point et que sa signature doit être exigée de tous les ecclésiastiques, dans des termes et des conditions sociaux et politiques.

Le jeu des puissances, des autorités, et la présence des pères, assez communs dans un discours de religion, prennent ici une forme et une signification toute singulière. Dans la lettre à la sœur, les diverses puissances sont citées, et la légitimité de leur action dans l'affaire du Formulaire soumise à une évaluation ${ }^{16}$. Quant à l'autorité, elle émane de certains pères. Les pères vivants des religieuses de Port-Royal, tout d'abord, et puis aussi d'autres, plus anciens.

Des propos de Saint-Cyran sont cités à deux reprises, saint Bernard l'est une fois. Saint Augustin est convoqué une fois, assez longuement, et d'une façon singulière puisque cette lettre le met en scène dans une fiction théorique :

"[11] Pour vous expliquer mieux ma pensée sur ces décisions du Saint-Siège, voici une comparaison qui me vient en l'esprit. Quoique tout le monde sache que le mystère de la Sainte Trinité est un des principaux points de notre foi, et que saint Augustin confesserait sans doute et signerait très librement, néanmoins si son pays était occupé par un prince infidèle qui voulût faire nier l'unité de Dieu et faire croire la pluralité des dieux, et que quelques-uns des fidèles, pour pacifier les troubles que cela exciterait, fissent un formulaire de foi sur ce point: "Je crois qu'il y a plusieurs personnes à

14. Constitutions du monastère de Port-Royal du Saint-Sacrement, édition présentée par Véronique Alemany, texte établi par Jean Lesaulnier, Nolin, coll. "Univers de Port-Royal ", Paris, 2004, p. 107.

15. Voir dans la lettre au père les références à Blaise et à Gilberte, frère et sœur, par le sang, de Jacqueline : " je le pouvais faire [fulminer] plus librement qu'un autre à cause de celui qui y a eu bonne part. " (Pascal, 1992 : 1092) ; "Je me remets entièrement à votre discrétion pour ces lettres. Mon inclination serait qu'elles ne fussent vues que de vous, mon Père, et de ma sœur Angélique. Néanmoins, si vous jugez à propos de les faire voir à M. de Gournay, vous le pouvez, mon Père. Ma sœur aussi est capable de les voir, et peut-être mon frère, s'il se porte bien. " (ibid. : 1093)

16. Pascal, 1992 : pp. 1082, 1086, 1088. 
qui l'on peut donner le nom de Dieu et leur rendre des adorations, etc.", sans autre explication, saint Augustin le signerait-il ? Certainement je ne le crois pas, et je crois encore moins qu'il le dût faire, quoique ce soit une vérité qu'il n'y a point de fidèle qui puisse mettre en doute; mais il ne serait pas le temps de le dire en cette manière. Vous ferez aisément le rapport de la comparaison.

" [12] On dira peut-être que notre autorité n'est pas du poids de celle de saint Augustin, et qu'elle est nulle. Je réponds à cela premièrement que je n'ai parlé de saint Augustin que par réponse à la seule que vous fîtes ces jours passés à tous mes doutes qui était que l'on se riait de nos craintes, et que saint Augustin signerait ce que nous craignions. Mais ce que je dis de saint Augustin, je le dis de vous et de moi, et des moindres personnes de l'Église; car le peu de poids de leur autorité ne les rend pas moins coupables s'ils [sic] l'emploient contre la vérité. » (Pascal, 1992 : p. 1087).

Cette fiction semble s'inscrire dans une pratique sociale plus ou moins largement partagée : fable contre fable, mais fiction plus conséquente puisque, au lieu de déplacer assez cavalièrement le père de l'Église dans le temps présent, elle imagine une situation dans laquelle Augustin, encore sans autorité, aurait été placé devant un choix analogue à celui qui est maintenant imposé aux ecclésiastiques de France. Non seulement le travail de transposition est plus rigoureux, mais il permet de débarrasser Augustin de son autorité, de la même manière que saint Pierre l'est, quelques ligne plus bas :

"Qui est le fidèle qui n'aurait point d'horreur de soi-même, s'il se pouvait faire qu'il se fût trouvé présent au conseil de Pilate où il aurait été question de condamner JésusChrist à la mort, s'il se fût contenté d'une manière d'opiner ambigüe par laquelle on eût pu croire qu'il était de l'avis de ceux qui le condamnaient, quoique en sa conscience, et selon son sens ses paroles tendissent à le délivrer ? Le péché de saint Pierre n'est-il pas infiniment moindre que ne serait une si extrême timidité ? Et cependant de quelle manière l'a-t-il regardé le reste de sa vie ? Et ce qui est bien considérable, c'est qu'encore qu'il fût destiné pour être le chef de l'Église, il ne l'était pas encore. Ce n'est donc que le péché d'un simple fidèle (...) » (Pascal, 1992 : 1087-1088).

Cette lettre use des plus grandes autorités de l'Église catholique pour déconstruire systématiquement l'autorité, et renvoyer les moindres personnes de l'Église à la responsabilité personnelle de leur propre conscience. C'est le sens de la citation de saint Bernard, dans laquelle l'emploi du féminin semble désigner précisément les religieuses plus que tout autre :

"Saint Bernard nous apprend, dans ses manières admirables de parler, que la moindre personne de l'Église, non seulement peut, mais doit crier de toutes ses forces lorsqu'elle voit les évêques et les pasteurs de l'Église dans l'état où nous les voyons, quand il dit: "Qui peut trouver mauvais que je crie, moi qui suis une petite brebis, pour tâcher d'éveiller mon pasteur que je vois endormi et prêt à être dévoré par une bête cruelle ?” Quand je serais assez ingrate pour ne le pas faire par l'amour que je lui porte et la reconnaissance que je lui dois, ne dois-je pas le faire par la crainte de mon propre péril ? Car qui me défendra quand mon pasteur sera dévoré ? »(Pascal, 1992 : 1085).

Non seulement, donc, les puissances souveraines de l'Église ou de l'État n'ontelles pas le droit d'exiger cette signature, mais de surcroît l'autorité est ici tant 
dénuée de force que c'est à la brebis de sauver le berger. Cet affaiblissement de l'autorité atteint jusqu'aux "pères » des religieuses de Port-Royal, dont on ne sait pas très bien, à plusieurs endroits, s'ils font ou non partie de ceux auxquels est reproché un excès de timidité (ibid., 1082) ${ }^{17}$, à tel point qu'il semble utile, à un certain moment de bien préciser par une dénégation qu'ils ne tombent pas sous le coup du reproche: "Ce que je ne dis pas pour nos Pères et pour nos amis ; je sais qu'ils ont autant d'horreur que moi des déguisements pour eux-mêmes ; mais je le dis pour l'état général où est l'Église. " (ibid., 1085). Le dispositif d'énonciation épistolaire accomplit fort exactement cette action de destitution d'une autorité en utilisant la relation d'autorité (lettre au père) pour développer une mise en écrit de la délibération d'une conscience comme correspondance avec un autre soi-même (lettre à la sœur).

C'est une affaire de conscience et l'action d'écriture d'une conscience, l'énonciation écrite en conscience, si écrire est permis, ne peut jamais, en tout état de cause, se réduire à signer une formule dont la raison d'être est de contraindre toutes les consciences à l'uniformité, à réduire toutes les consciences en une seule. L'écriture en conscience n'est pas une simple souscription, mais la production d'une nouvelle formule, la production de nouvelles formules, la mise en concurrence des formules dans une tentative pour trouver un accord sur une formule, qui se traduit par une individuation des formules. C'est ainsi que cette lettre (toujours la lettre à la sœur), répétant à sa façon le bredouillis des formules que nous venons de mettre en lumière par notre analyse de la Relation, propose quelques formules possibles (ibid., 1082) ${ }^{18}$ pour accompagner la signature et détruire ainsi l'uniformité du Formulaire. Et il est à noter que cela passe par la critique par une sœur du formulaire proposé par l'autre sœur et par son monastère: "L'autre chose que je vous réponds et que je vous avoue, ma très chère sœur, c'est que je n'ai pu jusques ici approuver entièrement votre formulaire tel qu'il est et que j’y voudrais quelque changement en quelques endroits. » (Pascal, 1992 : 1082).

Ainsi donc se dessine un conflit de radicalités, ou une montée en radicalité par le conflit. Sur les deux versants de la souscription, en deçà ou au-delà de la formule et de la signature, c'est un travail minutieux d'énonciation écrite qui produit ces radicalités. D’une part, dans la Relation des délibérations, une radicalité

17. Par exemple, p. 1082 : «Je ne puis plus dissimuler la douleur qui me perce jusques au fond du cœur de voir que les seules personnes à qui Dieu a confié sa vérité lui soient si infidèles, si je l'ose dire, que de n'avoir pas le courage de s'exposer à souffrir, quand ce devrait être la mort même, pour la confesser hautement. »

18. "Qui nous empêche, et qui empêche tous les ecclésiastiques qui connaissent la vérité, lorsqu'on leur présente le formulaire à signer, de répondre: "Je sais le respect que je dois à Messieurs les évêques; mais ma conscience ne me permet pas de signer qu'une chose est dans un livre où je ne l'ai pas vue, surtout tant de personnes habiles soutenant qu'elle n'y est pas" ; et après cela attendre en patience ou l'exil dont on menace les séculiers, ou la dispersion dont on menace les religieuses, les pertes, les prisons, et la mort si vous voulez ! "; voir également pp. 1085-1086, 1088-1089, 1090. 
d'institution dont la pointe est une formule écrite, infiniment reproductible à l'identique, supposée contraindre, et dans les faits contraignant presque toujours, à une profession de foi comme soumission uniforme à une doctrine, enseignement radical dans la mesure où il est progressivement défini comme celui qui, par fidélité aux traditions ecclésiastiques, tant dans son contenu que dans son mode d'élaboration, puise à la racine, c'est-à-dire dans la parole vivante du dieu fait homme, et dans l'Esprit. De l'autre part, dans les lettres dites « de Jacqueline Pascal ", c'est la racine individuelle de la foi chrétienne comme vérité, de chaque conscience comme témoin et lieu propre de cette vérité qui est recherchée. Le problème de la liberté de conscience dans une structure d'autorité et d'obéissance est ici résolu - mais à quel prix et de quelle façon ! - dans une action d'écriture qui consiste non pas seulement à écrire une lettre, ou deux lettres, ou un diptyque épistolaire, mais à aménager, dans le mouvement de l'énonciation, un lieu pour cette énonciation épistolaire tel qu'une multiplication de formules soit substituée à la simple souscription ou signature exigée et attendue. À la radicalité anti-dissidente produite par l'Église de France dans la Relation des délibérations s'oppose, sans ménagement ni médiation possible, une radicalité pleinement dissidente. Le lieu d'énonciation de la dissidence parfaite serait alors ce monastère bifide, lieu de la séparation, jusqu'à la "dispersion ", dans la clôture. Un travail de désaccord presque infini y est à l'œuvre, qui ne rencontre que deux limites. La première est celle qui sépare la dissidence de l'hérésie :

« [4] Mais peut-être on nous retranchera de l'Église ? Mais qui ne sait que personne n'en peut être retranché malgré soi, et que l'esprit de Jésus-Christ étant le lien qui unit ses membres à lui et entre eux, nous pouvons bien être privées des marques extérieures mais non jamais de l'effet de cette union, tant que nous conserverons la charité, sans laquelle nul n'est un membre vivant de ce saint corps ?" (Pascal, 1992: 1083).

La seconde limite que ce travail ne franchit pas est celle qui marque le point au-delà duquel la personne serait touchée dans l'intégrité de sa conviction. Ce n'est pas la radicalité d'un doute qui fonde le refus de la signature, mais au contraire celle d'une certitude. L'ignorance du fait, l'incapacité à pouvoir dire si les propositions condamnées par l'Église se trouvent bien ou ne se trouvent pas dans l'Augustinus, se convertissent en connaissance absolue de la vérité. Il est de l'ordre de la vérité de refuser de déclarer que telle erreur est dans tel livre si l'on n'a pas la certitude qu'elle y soit.

La possibilité d'une dissidence intérieure, non pas une de ces guerres intestines de Port-Royal dont nous venons de voir qu'elle sous-tendait toute la lettre mais, plus intimement encore, une séparation interne de l'auteur de la lettre, apparaît dans les troubles de la grâce, de ce qui, précisément, est tout à la fois l'enjeu du combat et le principe qui devrait guider ceux qui refusent le Formulaire. Il est surprenant de constater la rigueur de la répartition des mentions de la grâce entre les deux lettres. Dans la lettre à la sœur, qui pose les termes de la discussion et tente de proposer des actions, la grâce fait partie des matières du discours, en 
tant que sujet ultime de la polémique (Pascal, $1992: 1084)^{19}$, tandis que dans la lettre au père, qui explicite le problème des conditions de possibilité de cette mise en correspondance, elle figure comme premier principe et autorisation radicale de l'énonciation :

En vérité, mon Père, il semble que c'est un peu faire en cette matière comme ceux qui disent qu'on n'est pas obligé d'aimer Dieu, et qu'il suffit qu'on ne le haïsse pas. Mais si je me remets en discours, je n'en sortirai pas aisément. Pardonnez-le moi, mon Père, et ne croyez pas, je vous en supplie, quelque forte que je paraisse, que la nature n'appréhende beaucoup toutes les suites ; mais j'espère que la grâce me soutiendra, et il est vrai qu'il me semble quasi que je la sens. Je vous supplie très humblement, mon Père, de la demander pour moi. (Pascal, 1992 : 1093).

Bien certainement, l'expression de ce trouble fondamental signale-t-elle que, s'il n'est pas certain que la grâce soit avec l'auteur de cette lettre, à tout le moins il n'est pas non plus certain que le lieu de cette énonciation soit simplement naturel, et d'une nature déchue. Quoi qu'il en soit, l'incertitude sur la présence de la grâce, de même que celle qui entache la valeur de la première mission, qui toutes deux affectent la possibilité d'écrire ses pensées et de les adresser à quelqu'un, n'affaiblissent en aucune manière la conviction de connaître suffisamment la vérité pour mourir pour elle.

Nous rencontrons ici un point d'articulation décisif dans ce conflit de deux radicalités, la radicalité institutionnelle d'une Église, et la radicalité individuelle d'une conscience. Il se trouve que cette conscience, cette personne, comme nous avons été amenés à le constater à plusieurs reprises, est une des moindres brebis, ou tout au moins est-ce ainsi qu'elle peut se présenter : une religieuse, incapable donc, nécessairement, par définition dogmatique, puisqu'elle n'est ni docteur ni théologienne, de trancher dans une question aussi difficile que l'examen d'un traité sur la grâce. Elle n'est pas tout à fait la moindre personne de l'Église, puisqu'elle est capable, dans la représentation que les lettres qui lui sont attribuées donnent d'elle, d'écrire toutes ses pensées avec autant de force qu'elle le fait ${ }^{20}$. Ces deux lettres, tout en mettant en exergue la liberté individuelle d'une conscience, qui doit parvenir à déterminer quelle est la vérité, et à la défendre sans faillir, quel que soit le «poids » de son autorité, confèrent à une absence d'indépendance le rôle de condition nécessaire de cette liberté. C'est, comme le dit la proposition de formule que nous avons citée plus haut, l'avis de « tant de personnes habiles »

19. "Je crois que vous ne savez que trop qu'il ne s'agit pas ici seulement de la condamnation d'un saint évêque, mais que sa condamnation enferme formellement celle de la grâce de Jésus-Christ, et qu'ainsi, si notre siècle est si malheureux qu'il ne se trouve personne qui ose mourir pour défendre l'honneur d'un juste, c'est le comble de ne trouver personne qui le veuille pour la justice même. »; p. 1090 : « il m'est indifférent de quels termes on use, pourvu qu'on ne donne nul sujet de penser que nous condamnons ou la grâce de Jésus-Christ, ou celui qui l'a si divinement expliquée. "

20. Socialement, par sa position de pouvoir dans l'abbaye et par ses attaches familiales, l'auteur de ces lettres n'est pas le moindre des membres de l'Église catholique de France. 
qui justifie le refus de déclarer qu'une chose n'est pas dans un livre où « je » (le virtuel signataire de cet éventuel formulaire) avoue ne l'avoir " pas vue ». D'une façon plus significative encore, c'est l'ensemble de cette entrée en correspondance qui, dans ses procédures d'énonciation, produit cette dépendance, et cet effet de secte : références à la "chère mère » et à sa maladie, choix, comme destinataire de la lettre, d'un alter ego institutionnel, mentions de Jansénius et de Saint-Cyran, et mouvement de reconnaissance d'une autorité, celle d'Arnauld, entre les mains de qui cette mise par écrit des pensées est entièrement remise, abandonnée.

Aussi ces lettres, en représentant le problème de la souscription du Formulaire, et la tentative d'invention d'une solution à Port-Royal, donnent-elles existence à un certain type d'obéissance, et plus largement de dépendance aux autres, aux membres d'une famille spirituelle, dans une relation qui se présente comme parfaitement conforme aux règles, à un certaine forme de jeu d'autorité qui, contrairement aux organisations hiérarchiques des pouvoirs ecclésiastique et royal, ne construisent pas la société comme le lieu de la soumission, mais comme celui de la décision en conscience, et par conséquent du désaccord et de la dissidence permanents.

\author{
Alain CANTILLON \\ Paris III-Sorbonne Nouvelle \\ alain.cantillon@club-internet.fr
}

\title{
Bibliographie
}

BLET Pierre, 1995, Le Clergé du grand siècle en ses assemblées, Paris, Éditions du Cerf, pp. 222-224.

FraENKel Béatrice, 1992, La Signature, genèse d'un signe, Paris, Éditions Gallimard, coll. "Bibliothèque dans histoires".

GrÈs-GAYer Jacques, 2002, Le gallicanisme en Sorbonne, Chroniques de la Faculté de Théologie de Paris, (1657-1688), Paris, Honoré Champion, pp. 53-66.

Neveu Bruno, 1993, L'Erreur et son juge, remarques sur les censures doctrinales à l'époque moderne, Napoli, Bibliopolis, coll. "Serie Studi, Istituto italiano per gli Studi filosofici », 12.

PAsCAL Blaise, 1963, Euvres complètes, (prés. et notes de Louis Lafuma), Paris, Éditions du Seuil, coll. «L'Intégrale».

-, 1992, Euvres complètes, vol. IV, Paris, Desclée de Brouwer.

\section{Résumé}

Cet article analyse les relations entre radicalisme religieux et pratiques d'écritures autour des questions d'énonciation écrite posées par le fameux Formulaire. Il s'interroge sur la façon dont un livre, La Relation des délibérations du clergé de France, forme imprimée de pratiques d'écriture institutionnelles, produit et publie le Formulaire au sein de tentatives d'ensemble de définition et de saisie d'une nouvelle hérésie, le 
jansénisme. La Relation élabore la représentation d'un type de radicalité religieuse, que l'on peut qualifier d'institutionnelle. Cette étude ensuite s'interroge sur le passage de cette formule imprimée à une souscription, c'est-à-dire à un acte personnel d'écriture manuscrite. Deux lettres de Jacqueline Pascal, religieuse de Port-Royal (qui refusa de souscrire le Formulaire), opposent à la radicalité institutionnelle de la formule l'élaboration d'une autre radicalité religieuse dont la source est une conscience morale, dans sa singularité.

Mots-clés : énonciation écrite, Église catholique de France, gallicanisme, jansénisme, Port-royal, Jacqueline Pascal, formulaire.

\section{Abstract}

This article focuses upon the relationship between religious radicalism and writing practices with regard to problems of utterance arising out of the "Formulaire". First it examines the way a book, La Relation des délibérations du clergé de France, a printed form of institutional writing practices, produces and publishes the Formulaire within a global endeavor to grasp and define a new heresy called Jansenism. For these purposes La Relation elaborates the representation of a type of religious radicality that can be termed "institutional". Next, the essay questions how that printed formula was transformed by signing, that is to say by a personal act of hand-copying. Two letters by Jacqueline Pascal, a nun at the Port-Royal convent (who refused to sign the Formulaire), oppose the institutional radicality of the Formulaire with the elaboration of a religious radicality which emanates from the singularity of the moral conscience.

Key words: written utterance, Catholic Church of France, Gallicanism, Jansenism, Port-Royal, Jacqueline Pascal, formulaire.

\section{Resumen}

Este articulo analiza las relaciones entre radicalismo religioso y prácticas de escrituras alrededor de las cuestiones de enunciación escrita planteada por el famoso Formulario. Nos preguntaremos sobre la manera en que un libro. La Relation des délibérations du clergé de France, forma impresa de prácticas de escritura institucionales, produce y publica el Formulario dentro de intentos de conjunto de definición y de apropiación de una nueva herejía, el jansenismo. La Relation elabora la representación de un tipo de radicalidad religiosa, que se puede calificar de institucional. Este estudio luego se interroga sobre el pasaje de esta fórmula impresa a una suscripción, es decir a un acto personal de escritura manuscrita. Dos cartas a Jacqueline Pascal, religiosa de Port-Royal (que rechazó suscribir el Formulario), opone a la radicalidad institucional de la fórmula la elaboración de otra radicalidad religiosa cuya fuente es una consciencia moral, en su singularidad.

Palabras clave: enunciación escrita, Iglesia Católica de Francia, galicanismo, jansenismo, Port-Royal, Jacqueline Pascal, formulario. 